\title{
On the Usefulness of the Martensitic Transformation for the Evaluation of Phase Stabilities and Dislocation Properties in Long Range Ordered Alloys
}

\author{
M. Ahlers \\ Centro Atómico Bariloche and Instituto Balseiro, Comisión Nacional de Energía Atómica, 8400 Bariloche, \\ Argentina
}

\begin{abstract}
The small hysteresis in the martensitic transformations of shape memory alloys permits to determine experimentally differences in enthalpy and entropy to a high degree of precision. Due to the diffusionless nature of the martensitic transformation the configurational entropy remains invariant and the entropy difference results mainly from differences in the phonon spectra, which are accessible to experiment, èspecially the soft branches.
\end{abstract}

From measured enthalpy differences in the long range ordered alloys, those corresponding to the disordered state can be deduced which, together with the vibrational entropy differences, provide also valuable information on the equilibrium phases.

Close packed martensites with different stacking sequences are commonly observed, and from the critical transformation stresses their relative stability can be assessed. Split dislocations in long range ordered alloys with a stacking fault between them are similar to a thin martensite region, and therefore a comparison between both is of interest.

\section{THE TRANSFORMATION ENTROPY IN CU-Zn-AI ALLOYS AND ITS RELATIONSHIP WITH THE PHONON SPECTRA}

The $\mathrm{Cu}-\mathrm{Zn}-\mathrm{Al}$ alloys will be mainly discussed here, because the transformation entropy between the bcc type high temperature $\beta$ phase and the martensite has been studied in most detail for this alloy system. In addition, the entropy differences between the differently stacked close packed martensites are too small to be detected experimentally. This permits us to compare the vibrational entropy of the $\beta$ phase with that of the cubic fcc martensite, at least within the precision of the experiments.

The transformation entropy $\Delta S$ can be measured calorimetrically from the enthalpy of transformation, or mechanically from the temperature dependence of the critical stress to induce the martensite above $\mathrm{M}_{\mathbf{S}}$. Both methods have their limitations, since the $\Delta S$ are small, about 0.1 to $0.2 \mathrm{k}_{\mathrm{B}}$ ( $\mathrm{k}_{\mathrm{B}}$ the Boltzmann constant), but an uncertainty of $\pm 10 \%$ in the measured values seems to be a reasonable guess [1]. The $\Delta \mathrm{S}$ cannot be due to differences in the Debye temperature between the structures, but must be related to contributions from soft phonons which are fully excited already at $80 \mathrm{~K}$. This is deduced from the fact that the critical stress to induce the martensite increases linearly with temperature, which according to the Clausius-Clapeyron equation implies that $\Delta S$ is constant [2]. The soft phonons which contribute mainly to $\Delta \mathrm{S}$ are thought to be those belonging to the $\mathrm{TA}_{2}$ transversal $\left\{\xi \xi_{0}\right\}\langle\xi \bar{\xi} 0\rangle$ branch of the $\boldsymbol{\beta}$ phase, or being close to them. This is supported by neutron measurements [3] which show that this branch is of 
sufficiently low energy to be fully excited already at $80 \mathrm{~K}$. Moreover, sound velocity measurements indicate that the phonons at low wave vectors $\mathrm{k}$ in the $\mathrm{TA}_{2}$ branch have considerably lower energy than those propagating normal to the habit plane in the martensite [3]. It has therefore been suggested that a small fraction $\mathrm{p}$ of all phonons near the $\mathrm{TA}_{2}$ branch in the $\beta$ phase are responsible for $\Delta \mathrm{S}[2,5,6]$. A p of $4 \%$ has been estimated, which is a reasonable quantity. It is highly desired, however, to obtain the $p$ directly from the phonon spectrum of the bcc phase, in order to put the supposition that the martensite structure is not affecting greatly $\Delta \mathrm{S}$ on firmer ground. It should only be mentioned here, that in an fcc 6R martensite at $\mathrm{e} / \mathrm{a}=1.53$ a strong stress dependence of the lattice parameters has been observed [7], which corresponds to a lower elastic constant, $c^{\prime}$, than that expected for the fcc lattice. Whether this is an indication that also in the fcc martensite softening of the phonons occurs and how it modifies $\Delta S$ is not clear at this moment.

In the $\mathrm{Cu}-\mathrm{Zn}-\mathrm{Al}$ alloys $\Delta \mathrm{S}$ has been found experimentally to increase with $\mathrm{e} / \mathrm{a}$ according to [8]:

$$
\Delta S=(0.51 e / a-0.58) k_{B}
$$

A composition dependence of $\Delta S$ at fixed e/a could not be detected experimentally. This is not surprising since the $\mathrm{M}_{S}$ temperature can be measured only in a small composition range for a given e/a, which is too small to notice shifts in $\Delta \mathrm{S}$. On the other hand the alloys with a fixed $\mathrm{MS}_{\mathrm{S}}$ cover a considerably larger composition (and e/a) range which extends from binary $\mathrm{Cu}-\mathrm{Zn}$ to binary $\mathrm{Cu}-\mathrm{Al}$.

It seems surprising that $\Delta S$ increases with e/a. The opposite would have been expected, since a higher $\beta$ phase stability implies smaller vibrational amplitudes and thus an increase also in the elastic constant $c^{\prime}$. For binary $\beta \mathrm{Cu}-\mathrm{Zn}$ it has in fact been shown that the Debye temperature augments with e/a [9], as should be. However, the fallacy of this argument lies in the fact that the $\Delta \mathrm{S}$ as a function of e/a is not measured at constant $T$, but when the enthalpies of formation of the $\beta$ phase and the martensite are equal (at $\mathrm{MS}_{\mathrm{S}}=0 \mathrm{~K}$ ), or nearly so.

It has been suggested [2] that $\Delta S$ is related to $c^{\prime}$ at the $M_{S}$ temperature: When the k dependence of the frequency for the $\mathrm{TA}_{2}$ branch is sinusoidal, the slope at small $\mathrm{k}$, which determines $c^{\prime}$, and the frequencies near the Brillouin zone boundary, which contribute most to $\Delta S$, are related. It has been shown in fact [5], that $c^{\prime}$ near $\mathrm{M}_{\mathrm{S}}$ is the same in $\mathrm{Cu}-\mathrm{Al}-\mathrm{Be}$ alloys of different composition. For $\mathrm{Cu}-\mathrm{Zn}-\mathrm{Al}$ alloys not enough measurements for $c^{\prime}$ exist at $M_{S}$. Therefore it has been attempted to extrapolate measured $c^{\prime}$ to $M_{S}$, in order to check whether $\Delta S$ and $c^{\prime}$ can be correlated [6]. It is found, however, that $c^{\prime}$ increases with e/a, instead of decreasing, necessary to explain the changes in $\Delta S$ [6]. It has been speculated [6] that a softening of the phonon modes near $\mathrm{TA}_{2}$ occurs for large $\mathrm{k}$, and that this softening increases with e/a. Precursor softening of some vibrational modes prior to the martensitic transformation has often been observed, but whether it depends on e/a is not known, but could be checked by measuring the phonon spectra at different e/a.

In the $\mathrm{Cu}-\mathrm{Zn}$ - $\mathrm{Al}$ system the entropy difference between the differently stacked close packed martensites is too small to be measured [10]. This need not always be the case. For example, in Ni-Al a pronounced temperature dependence of the critical transformation stress has been found not only for the transformation from $\beta$ to $7 \mathrm{R}$, but also for that between $7 \mathrm{R}$ and $3 \mathrm{R}$ [11]. For these alloys $\Delta S$ is clearly not solely determined by the vibrational modes in the $\beta$ phase. However, the $7 \mathrm{R}$ has a strong monoclinic distortion, and $3 R$ has a structure intermediate between the bcc and the fcc lattice, contrary to the results for the corresponding $6 \mathrm{R} \mathrm{fcc}$ martensite in the $\mathrm{Cu}-\mathrm{Zn}-\mathrm{Al}$ alloys.

The $\Delta S$ for the martensitic transformation are generally measured at temperatures below $100^{\circ} \mathrm{C}$. It would be desired to extrapolate $\Delta S$ to higher temperatures, in order to obtain information of the relative stability of equilibrium phases at high temperatures, where short range or long range order contributions can be neglected. With increasing temperature the anharmonicity comes into play. Since, however, no anharmonic effects have been found by neutron diffraction in $\beta$ phase CuZnAl at room temperature [12], 
it is not expected that $\Delta S$ changes strongly with temperature. In fact, measured entropy and enthalpy differences for the martensitic transformation can account well for the stability ranges of the $\alpha$ and $\beta$ phases at high temperatures [13], at least as a first approximation.

\section{TRANSFORMATION ENTHALPIES AND THEIR RELATIONSHIP WITH THE STABILITY OF THE EQUILIBRIUM PHASES}

The enthalpy differences between the high temperature $\beta$ phase and the $9 \mathrm{R}$ or $18 \mathrm{R}$ martensites in the $\mathrm{Cu}-$ $\mathrm{Zn}$-Al system have been measured in a wide composition range [8]. In addition, those between the different martensite phases $6 \mathrm{R}$ (or 3R), $18 \mathrm{R}$ (or 9R) and $2 \mathrm{H}$ are known [10]. The $6 \mathrm{R}$ (or 3R) martensite is cubic, based on the fcc lattice in the fully transformed state [7]. It is thus possible to compare energetically an fcc with a bcc based long range ordered lattice which have the same atom distribution, since the martensitic transformation does not permit rearrangements of atoms on the lattice. Another advantage of the martensitic transformation is that it occurs at low temperatures, at which the high temperature phase has attained already its maximum degree of long range order, eventually by an additional adequate heat treatment [13]. The energy of the perfectly ordered state depends only on composition and on the pair interchange energies. Since the martensitic transformations in the $\mathrm{Cu}-\mathrm{Zn}-\mathrm{Al}$ system are observed in a rather small e/a range from 1.4 to 1.5 approximately, the neglect of the composition dependence of the pair interchange energies does not introduce major errors in the evaluation. For the description of long range order it is often sufficient to include only first and second neighbor interchange energies.

The pair interchange energies can be determined experimentally from critical ordering temperatures, from short range order measurements or by comparing martensitic transformations with different types of long range order. In $\mathrm{Cu}-\mathrm{Zn}-\mathrm{A} 1$ a $\mathrm{B} 2$ or an $\mathrm{L} 21$ ordered phase can be transformed $[8,14,15]$. By subrracting from the measured transformation enthalpy the contribution from the long range order it is possible to obtain the enthalpy difference between the disordered fcc and bcc phases. These phases are equilibrium phases at high temperatures, and their enthalpies of formation determine the stability range in the phase diagram. By the traditional methods it is difficult to determine their enthalpy differences, since they are small. For this reason the evaluation of martensitic transformations presents an important alternative method to obtain results also for the equilibrium phases with the required precision.

It is now possible to calculate enthalpies from first principles. The validity of our approach is underlined by the fact that for binary $\mathrm{Cu}-\mathrm{Zn}$, calculated and experimentally determined enthalpy differences between the $\alpha$ and $\beta$ phases agree closely [16].

We have presented here only the discussion for the $\mathrm{Cu}-\mathrm{Zn}-\mathrm{Al}$, in order to show the usefulness of the study of martensitic transformations for a better evaluation and understanding of the factors which determine the equilibrium phases. We have not discussed here the relationships between bcc and hexagonal, nor $\mathrm{fcc}$ and hex equilibrium phases [14]. Similar martensitic transformations have also been found in other noble metal alloys, and can be used to discuss the behavior of the noble metal alloy phases [14], in order to understand better the reason why the electron concentration plays such an important role in their stability.

\section{DISLOCATIONS IN LONG RANGE ORDERED ALLOYS AND THEIR RELATIONSHIP WITH THE MARTENSITIC TRANSFORMATION}

Long range ordered intermetallic compounds are now widely studied due to their often favorable mechanical behavior at elevated temperatures. A typical example is the $\mathrm{Ni}_{3} \mathrm{Al}$ superalloy. In the $\mathrm{Ni}-\mathrm{Al}$ system also a martensitic transformation from the $\mathrm{B} 2$ ordered high temperature to a $7 \mathrm{R}$ martensite has been observed around $37 \mathrm{at} \% \mathrm{Al}[17]$. In the following we want to point out that the study of the 
martensitic transformation can be useful for a better and more quantitative understanding of the structure and mobility of the superdislocations in the ordered phase.

In the $\mathrm{Ni}_{3} \mathrm{Al}_{1} \mathrm{Ll}_{2}$ long range ordered $\mathrm{Cu}_{3} \mathrm{Au}$ type structure, the superdislocations are split into two pairs with an antiphase boundary between them. Each of the two pairs consists of two partial dislocations with a stacking fault betweer them. The stacking faults are often considered as a thin hexagonal layer embedded in the face centered matrix. In the $\mathrm{Cu}_{3} \mathrm{Au}$ structure it has in addition a different, less favorable order distribution due to the diffusionless glide processes, and is called a complex stacking fault (CSF).

The martensitic transformation proceeds from a $\mathrm{B} 2$ ordered to an $\mathrm{L} 1_{\mathrm{O}}$ ordered face centered structure, containing regularly spaced stacking faults which lead to the $7 \mathrm{R}$ structure. In contrast to the stacking faults in Ni3 Al, the order due to first and second neighbors is not changed. By applying a stress the stacking faults can be eliminated and $3 R$ results. If the $7 R$ martensite can be considered energetically as a face centered lattice with stacking faults which do not or only weakly interact between each other, then from the transformation stress $7 R$ to $3 R$ the energy per fault can be determined experimentally. Its extrapolation to the Ni3 $\mathrm{Al}$ alloy with $25 \mathrm{at} \% \mathrm{Al}$ would then permit to separate its stacking fault energy into an order contribution and a superlattice intrinsic stacking fault (SISF) contribution. As yet there are not sufficient data available to perform this extrapolation from $37 \mathrm{at} \% \mathrm{Al}$ to $25 \mathrm{at} \% \mathrm{Al}$. There are several other factors which have to be taken into account when the dislocation structure is to be compared with the characteristics of the martensitic transformation in the $\mathrm{Ni}-\mathrm{Al}$ alloys. The transformation stress from $7 \mathrm{R}$ to $3 \mathrm{R}$ is strongly temperature dependent, which shows that the vibrational entropy is different in both phases [11]. This should not be surprising, since $3 \mathrm{R}$ has a large tetragonal distortion which places it in the middle between an fcc and bcc ordered lattice [17], and thus small displacements towards the bcc structure do not encounter such high activation barriers compared to paths from fcc to bcc. In the $\mathrm{Cu}$ $\mathrm{Zn}$-Al the $18 \mathrm{R}$ martensite remains closer to the orthorhombic structure and the entropy difference between $6 \mathrm{R}$ and $18 \mathrm{R}$ is so small that it cannot be measured [10]. Ni3 Al is cubic, but nevertheless it would be interesting to know whether the stacking faults are stabilized by the vibrational entropy.

A second point to discuss is the relationship between the energy of the stacking faults leading to $7 \mathrm{R}$ and that of a single fault in 3R: In Cu-Zn-Al it has been found [10] that the stacking fault energy in $18 \mathrm{R}$ and $2 \mathrm{H}$ are strongly composition dependent, considering energetically the $18 \mathrm{R}$ and $2 \mathrm{H}$ as a face centered lattice with stacking faults on each third or second close packed plane. Their difference, however, is constant in the reduced composition range, in which transformations are observed, and amounts to 11 $\mathrm{mJ} / \mathrm{m}^{2}[8]$, which is small and which indicates that in $18 \mathrm{R}$ with the more widely spaced faults the interaction is less, so that the fault energy in $18 \mathrm{R}$ should not be very different from that of a single fault in a fcc inatrix. For Ni-Al a similar information does not exist, but the observation of irregularly spaced stacking faults in $7 R$ is an indication that the free energy of a single fault and that in $7 R$ is not very different.

A third factor of importance in the evaluation of the martensite structures are the changes in lattice spacing. Although the atomic volume changes very little during the martensitic transformation lattice distortions are often observed. In the $\mathrm{Cu}-\mathrm{Zn}-\mathrm{Al}$ alloys the $6 \mathrm{R}$ phase is cubic, but $18 \mathrm{R}$ shows a monoclinis distortion which is related to the order energy [7]. In Ni-Al the $3 \mathrm{R}$ martensite is already setragonaly distorted, and $7 R$ seems to have a similar distortion of the basal plane, but high precision lattice parameter measurements are clearly required. It shall only be pointed out that a stacking fault in Ni3Al may like to relax to slightly different interatomic distances. This question can be of importance at higher temperatures at which sufficient thermal energy is available to produce the relaxation and immobilize the dislocations.

The martersitic transformation and the movement of partial dislocations are both diffusionless processes, they lead to structures which are unstable when diffusional processes are permitted. The $\mathrm{Cu}-\mathrm{Zn}-\mathrm{Al}$ 
martensite gets stabilized by a change in the degree of long range order [7] and by a decomposition into different phases at higher temperatures. Similar processes can also modify the dislocation structure and mobility: A dislocation which moves sufficiently slowly or gets immobilized in front of an obstacle can lower its energy if diffusional processes at the dislocation core are possible. Due to strong lattice distortions near the center of the dislocations it can be anticipated that relaxation and diffusional processes are easier near the dislocation than in the bulk, and therefore can occur at lower temperatures.

In the $\mathrm{Ni}_{3} \mathrm{Al}$ alloys the plastic behavior at elevated temperatures is of interest. The typical dislocation configurations are the Kear-Wilsdorf locks in which the two dislocation pairs are separated by an antiphase boundary on the (001) plane. These configurations become mobile only when they transform to a higher energy planar defect. In situ experiments have shown [18] that the dislocation movement is jerky, interrupted by intervals during which the dislocations become immobile. In these intervals they become susceptible to relaxation processes, if sufficiently fast. The question whether diffusional processes play any role in the positive temperature dependence of the critical stress remains open, according to our view. It is generally difficult to measure slow diffusion processes. Here again martensitic transformations can help, since diffusion very sensitively manifests itself in a change of the $\mathrm{M}_{\mathrm{S}}$ temperature $[7,19]$.

The aim of this section is to show that there exists a close relationship between the martensitic transformation and the mobility of partial dislocations. It is hoped that the combination of both phenomena wiil lead to new and interesting results.

\section{REFERENCES}

[1] A. Planes, R. Romero and M. Ahlers, Scripta Met. 23 (1989) 989.

[ 2] R. Romero and M. Ahlers, J. Phys: Condens. Matter 1 (1989) 3191.

[3] G. Guénin, D. Rios-Jara, Y. Murakami, L. Delaey and P.F. Gobin, Scripta Met. 13 (1979) 289.

[4] G. Guénin, D. Rios-Jara, M. Morin, L. Delaey, R. Pynn and P.F. Gobin, J. Physique Coll. 43, C4 (1982) 597.

[5] A. Planes, Ll. Mañosa, D. Rios-Jara and J. Ortin, Phys. Rev. B45 (1992) 7633.

[6] R. Romero and J.L. Pelegrina, Phys. Rev., accepted.

[7] F. Saule and M. Ahlers, Subm. Acta Metall. Mater. (1994).

[ 8] J.L. Pelegrina and M. Ahlers, Acta Metall. Mater. 40 (1992) 3205.

[9] B.W. Vea! and J.A. Rayne, Phys. Rev. 128 (1962) 551.

[10] M. Ahlers and J.L. Pelegrina, Acta Metall. Mater. 40 (1992) 3213.

[11] K. Enami, M. Togawa and V.V. Martynov, Proc. IUMRS-93 in press.

[12] V. Gillete and J.R. Granada, Discussion Meeting on Cu-Zn-Al martensite, Report R84-1984,

K.U. Leuven, Belgium.

[13] A. Planes, R. Romero and M. Ahlers, Acta Metall. Mater. 38 (1990) 757.

[14] M. Ahlers, I. Phys: Condens. Matter 5 (1993) 8129.

[15] M. Ablers, Phil Mag.A. 70 (1994) 247.

[16] P.E.A. Turchi, M. Sluiter, F.J. Pinski, D.D. Johnson, D.M. Nicholson, G.M. Stokes and J.B. Staunton, Phys. Rev. Letters 67 (1991) 1779.

[17] S. Chakravorty and C.M. Wayman, Mat.Trans. 7A (1976) 555.

[18] D. Caillard, A. Couret, N. Clément, S. Farenc, and G. Molenat, "Strength of Metals and Alloys", eds. D.G. Brandon, R. Chaim and A. Rosen, (Freund Publ. House, London 1991) pp. 139.

[19] A. Abu Arab and M. Ahlers, Acta Metall. 36 ( 1988) 2627. 